DOE/PC/94120--TH

\title{
QUARTERLY REPORT NO. $4^{*}$
}

\author{
Investigation of the Effect of Coal Particle Sizes on the Interfacial and \\ Rheological Properties of Coal-Water Slurry Fuels
}

CONTRACT NO.: $\quad$ DE-FG22-94PC94120

$\begin{array}{ll}\text { TECHNICAL MONITOR: } & \text { Dr. Soung S. Kim } \\ & \text { DOE-PETC, Pittsburgh, Pennsylvania }\end{array}$

REPORT PERIOD: $\quad$ 04/01/95-06/30/95

PRINCIPAL INVESTIGATOR: Ken D. Kihm, Associate Professor Department of Mechanical Engineering Texas A\&M University College Station, TX 77843-3123

Tel. (409) 845-2143 and Fax. (409) 862-2418

(*This report also has been submitted for the
Contractors Meeting held in Pitsburgh.)
work sponsored by an agency of the United States
implient, or assumes any legal liability or responsi-

This report was prepared as an account of work sponsored by an agency of the United States employees, makes any warranty, express or implied, or assumes any legal liability or responsibility for the accuracy, completeness, or usefulness of any information, apparatus, product, or process disclosed, or represents that its use would not infringe privately owned rights. Reference herein to any specific commercial product, process, or service by trade name, trademark, manufacturer, or otherwise does not necessarily constitute or imply its endorsement, recommendation, or favoring by the United States Government or any agency thereof. The views and opinions of authors expressed herein do not necessarily state or reflect those of the United States Government or any agency thereof. 


\title{
ON THE INTERFACIAL AND RHEOLOGICAL PROPERTIES
}

\author{
OF COAL-WATER SLURRY FUELS
}

\author{
KEN D. KIHM, ASSOCIATE PROFESSOR \\ PAUL DEIGNAN, RESEARCH ASSISTANT \\ DEPARTMENT OF MECHANICAL ENGINEERING \\ TEXAS A\&M UNIVERSITY \\ COLLEGE STATION, TX 77843-3123
}

\begin{abstract}
Experiments were conducted to investigate the effect of particle size on coal-water slurry (CWS) surface tension properties. Two different coal powder samples of different size ranges were obtained through sieving of coal from the Upper Elkhorn Seam. The surfactant (anionic DDBS-soft, dodecylbenzene sulfonic acid) concentration varied from 0 to $1.0 \%$ in weight while the coal loading remained at $40 \%$ in weight for all the cases. A du Nouy ring tensiometer and a maximum bubble pressure tensiometer measured the static and dynamic surface tensions, respectively. The results show that both static and dynamic surface tensions tend to increase with decreasing coal particle sizes suspended in CWS fuels. Examination of the peak pressure, minimum pressure, surfactant diffusion time, and dead time were also made to correlate these microscopic pressure behavior with the macroscopic dynamic surface tension and to examine the accuracy of the experiment.
\end{abstract}

\section{INTRODUCTION}

Coal-water slurry atomization involves interactions between three different phases: solid (coal particles), liquid (water and additives), and gas (air or steam). Surface or interfacial tension is one of the significant properties in determining atomization characteristics of liquid or slurry fuels. Under the quasi-equilibrium conditions of low shear-rates of excessively slow atomization, the static surface tension of the fluid is an appropriate measure of the fluid's ability to form small radius droplets. However, the surfactants, additives or wetting agents presented in the CWS formulation do not reach an equilibrium concentration throughout the solid-liquid or liquid-vapor interfaces at higher shear rates. Therefore, dynamic surface tension should be a more appropriate measure in assessing the atomization that usually occurs at high shear rates.

The dynamic surface tension of a fluid is simply a measurement of the surface tension at a particular rate of surface formation or shear rate. The static and dynamic surface tension values are the same for pure fluids, such as water. The values of dynamic surface tension for slurry mixtures containing solid particles and various additives may be much higher than the corresponding static surface tension because insufficient time exists for the migration of surfactant additives to the atomized interface from the bulk mixture. The difference between the two surface tension values increases at higher shear rates that allows less time for the surfactant migration.

It is the intention of the present work to examine the effect of coal particle sizes on CWS static and dynamic surface tension properties. Rheology shows that the slurry viscosity generally increases with decreasing mean particle size [1]. When particles are suspended in the solution dispersed with additives and/or solvents, adsorption or solvation layers are formed on the particle surface which increases the effective volume ( $f$ ) of the particle. This effective volume increase is particularly significant for small particles which can explain the increase of the slurry viscosity with 
decreasing mean particle size. To the extent of our literature survey, no such correlation for surface tension, whether static or dynamic, has been published. Examination of particle size effect on interfacial properties of CWS fuels is attempted using specially prepared coal particle samples.

\section{EXPERIMENTAL PROCEDURES}

\section{Maximum Bubble Pressure Technique}

Figure 1 illustrates the operating principle of the maximum bubble pressure tensiometer technique for dynamic surface tension measurement [2]. The CWS sample was contained in a vessel into which is inserted a specially designed capillary tube with a small outlet diameter of one millimeter. As bubbles were formed, grew, and detached from the capillary tube orifice, variations of the bubble pressure occurred because of the change in bubble radius. For each bubble, maximum pressure was recorded when the bubble radius reached its minimum at the orifice radius. For different bubble generation frequencies, the dynamic surface tension was calculated from the measured maximum bubble pressure substituted into a simple relation

$$
\sigma=\left(p_{\max }-\rho g h\right) r_{c} / 2
$$

where $\rho$ is the sample fluid (CWS) density, $g$ is the gravitational acceleration, $h$ is the height above the capillary outlet, and $r_{c}$ is the radius of the capillary outlet. The maximum bubble pressure, $p_{\text {max }}$, corresponds to the inside air pressure when the radius of the growing bubble is equal to the capillary radius, $r_{c}$. The KRUSS Model BP1 tensiometer was adopted to measure dynamic surface tension of CWS fuels. The accuracy of the tensiometer was tested by measuring surface tension for distilled and deionized water. The result ensured a satisfactory measurement accuracy showing less than $2 \%$ deviations from the surface tension values listed in the CRC Handbook [3].

\section{Modification of the Existing Kruss Tensiometer}

Analog signal output from the diaphragm type pressure transducer of the Kruss tensiometer is interfaced with an A-to-D board installed in an 386 PC. ATLAB data acquisition software from Data Translation Co. samples the analog signal at a rate of $0.2 \mathrm{kHz}$ and digitizes them into the computer hard disk memory. One batch of data scanning over the specified bubble frequency range from one to ten per second requires approximately $1 \mathrm{MB}$ hard disk memory capacity. A simple FORTRAN program identifies the peak voltage, minimum voltage, bubble life time, and dead time.

Figure 2 shows a temporal history of voltage readings for a typical case of $1 \%$ DDBS-soft aqueous solution. One cycle period of the curve is equivalent to the bubble life time, or the reciprocal of the bubble frequency. The bubble pressure increases as the nitrogen gas inflow makes the bubble grow and the bubble diameter decreases from the infinitely large curvature of the initially flat interface at the orifice exit. The bubble pressure increase as the bubble diameter decreases with time. The bubble pressure increases at a faster rate when a surfactant acts to reduce the surface "holding" tension. While the bubble pressure increases, the surfactant migrates to the newly created bubble surfaces. The surface is 'aged' with surfactant diffusion during the period of ascending curve, which is now called 'Diffusion time'. The bubble pressure reaches its maximum value (peak voltage) when the bubble grows to the orifice diameter.

Further growth of the bubble beyond the peak voltage increases the bubble diameter and the bubble pressure decreases first smoothly, and then the descending pressure curve becomes irregular most probably because of the geometrical distortion of the enlarged bubble. The enlarged and distorted bubble detaches from the orifice and the cycle completes at the time of the minimum voltage reading. The duration from the peak till the cycle completion does not directly influence the measurement of the maximum bubble pressure, and this time period is now called 'Dead time'. 
Coarsely ground Upper Elkhorn Seam coal provided by DOE-PETC was classified into several different size ranges using a sieve shaker. To minimize the coal oxidation during the sieving, the sieve array was sealed with tape and the duration of sieve shaking was kept at a minimal necessary level. In most cases, the sieving was completed within thirty minutes. Two samples containing the largest and the finest particles were selected for testing so the effect of particle size on surface tension values could be distinctively observed. The coarse sample contains coal particles in the range of 180 to $250 \mu \mathrm{m}$, and the fine sample contains coal particles less than 63 $\mu \mathrm{m}$. The present report presents results for only DDBS-soft surfactant which shows the best performance among the tested five surfactants [4]

The coal and water was completely mixed by a rotating mixer running for twelve to twenty-four hours. The specified amount of surfactant was then added and mixed by a magnetic stirrer for 5 minutes. All the present experiment used $40 \%$ weight CWS fuels. The slurry viscosity increases with increasing ratio of the packing density which is defined as the ratio of the solid volume fraction to the maximum attainable solid volume fraction, i.e., $\mathrm{f} / \mathrm{f}_{\mathrm{m}}[1]$. The relatively uniform size distribution of the sieved coal particles tends to reduce the maximum attainable solid volume fraction and increases the packing density compared with coal powder of a wide size distribution. $50 \%$ or higher weight CWS fuel samples were too viscous and the tensiometers were not able to function with acceptable accuracy.

\section{RESULTS AND DISCUSSION}

\section{Static Surface Tension}

A du Nouy ring tensiometer measured static surface tension for the coarse and fine CWS samples mixed with DDBS-soft surfactant in Fig. 3. The static surface tension decreases with increasing surfactant concentration and approaches a saturated value beyond a certain surfactant concentration. Before reaching this certain concentration, which is called a critical micelle concentration (CMC), the fine CWS sample shows larger surface tension values than the coarse sample and the CMC of the fine CWS is higher than the coarse CWS.

The dashed arrows indicate the CMC of the fine sample, the solid arrows represent the CMC of the coarse sample, and the empty arrows are for the CMC of an aqueous solution of the specified surfactant. The CMC values for CWS fuels are higher than their aqueous counterparts. The primary reason for this is believed to be the surfactant adsorption on the coal particle surfaces which requires more amount of surfactant than the aqueous solution (Fig. 4). The higher CMC of the fine CWS compared with the coarse CWS can also be explained by the surface adsorption. Smaller coal particles create more total surface area than larger particles for the same coal loading, which causes the overall surface adsorption of surfactant to increase. This needs higher surfactant concentration for the saturated surface tension level and higher CMC.

\section{Dynamic Surface Tension}

Figure 5 shows dynamic surface tension versus bubble frequency for the two selected CWS samples of $40 \%$ coal weight containing DDBS-soft surfactant. The family of curves in each plot, from the top to the bottom, correspond to $0,0.1 \%, 0.5 \%$ and $1.0 \%$ concentrations. At each surfactant concentration, the fine CWS fuel shows consistently higher dynamic surface tension values than the coarse CWS under the same bubble frequency. Two reasons can be listed for the distinction: (1) smaller coal particles of higher number density contained in the fine CWS sample 
enhance the physical blockings against the surfactant migration to the bubble-created surface and reduce the surfactant diffusion into the bubble surface (Fig. 4), and (2) the increased total particle surface areas of the fine CWS sample increase the surfactant adsorption.

For the case with no surfactant mixed, the top curve in each plot, the higher surface tension for the fine CWS is attributed to the different bubble surface characteristics depending on the particle size. When the surfactant concentrations are zero or close to the static $\mathrm{CMC}$ level of $0.1 \%$, the dynamic surface tension is nearly independent of the bubble frequency, which shows that the dynamic effect on surface tension is not pronounced for low surfactant concentrations. With increasing surfactant concentration, however, the dynamic surface tension shows a gradual increase with increasing bubble frequency, as the increased bubble frequency does not allow sufficient migration time for the surfactant.

\section{Peak Voltage and Minimum Voltage}

Figures 6-a and b show the temporal history of peak and minimum voltage readings for the fine and coarse CWS samples, respectively. The peak voltage data directly convert to evaluate the dynamic surface tension values. The peak voltage data obtained for the fine sample (Fig. 6-a) and the coarse sample (Fig. 6-b) show close similarity to the dynamic surface tension curves presented in Figs. 4-a and b, respectively. The higher dynamic surface tension of the fine CWS sample, which has been discussed in depth in the previous section, explains the higher peak voltage and the relatively lower dynamic surface tension of the coarse CWS results in the lower peak voltage. this finding is consistent regardless whether the surfactant is presented (1.0\%) or not.

The nearly constant minimum voltage over the range of bubble frequencies at a given surfactant concentration shows that the system recovers to an identical initial condition before beginning of the next cycle. This also ensures the accuracy of the dynamic surface tension measurement that assumes an identical initial condition regardless of the bubble frequency. The minimum voltage for the coarse sample gradually varies with the bubble frequency range, but not showing any particular temporal correlation. This variation is attributed to the bubble pressure detection uncertainties associated with the presence and movement of relatively large coal particles at the gas-liquid interface.

The minimum voltage represents the hydrostatic pressure at the depth of the interface at the time of cycle completion or of new cycle initiation. The minimum voltage can decrease when the gasliquid interface recedes from the orifice exit and stays inside the orifice at the time of cycle completion. The receding of the gas-liquid interface is likely to occur at higher surfactant concentration that enhances the solution wetting and increases the interface penetration inside the orifice. Results for both fine and coarse samples (Figs. 6-a and b) show that the minimum voltage level decreases with increasing surfactant concentration.

Increase in the minimum voltage could occur when the hydrostatic pressure is higher than that at the orifice exit. This may occur as a result of an incomplete bubble detachment at the end of the cycle. The remaining undetached portion of the bubble can result in a deeper initial depth than the orifice exit and the pressure transducer will detect a higher hydrostatic pressure than it should be. This type of bias may occur at extremely high bubble frequencies where the dead time is insufficient for full recovery of the cycle with a complete bubble detachment. A high-speed synchronized cinematograph could be employed to carefully visualize the bubble growth and detachment as a future investigation. 
Figures $7-a$ and $b$ show the percent dead time over the bubble life time as a function of the bubble frequency for the fine and coarse CWS samples with or without surfactant. This ratio increases linearly with increasing bubble frequency and also increases with increasing surfactant concentration. Reduced surface tension at higher surfactant concentrations expedites the bubble growth rate and shorten the diffusion time. The dead time, which occupies the remainder of the bubble life time, increases and the percent dead time goes up with increasing surfactant concentration for a given bubble life time.

For a fixed surfactant concentration the dead time ratio increases with the bubble frequency. This implies that the percent diffusion time decreases with increasing bubble frequencies and the surface aging by the surfactant migration is insufficiently achieved. This relatively short diffusion time at higher bubble frequencies retards a full benefit of surfactant. Larger dynamic surface tension values measured at higher bubble frequencies (see Figs. 4-a and b, for example) are attributed to the shortened diffusion time with increasing bubble frequency.

\section{CONCLUSION}

The effects of coal particle size on the CWS interfacial properties by measuring the dynamic surface tension using a Kruss tensiometer and by examining the detailed bubble pressure variation with time. Fundamental conclusions reached are:

1) Examination of interfacial properties of CWS fuel samples of different coal particle size ranges shows that the CWS static and dynamic surface tensions tend to increase with decreasing coal particle sizes. This finding consists with the measured peak voltages, which converts into the dynamic surface tension.

2) For the case of coarse CWS sample, the relatively larger coal particles cause the minimum voltage to slightly fluctuating, but remaining nearly constant through the range of bubble frequencies considered. The minimum voltage remains constant for the fine CWS sample.

3) The dead time ratio over the bubble life time increases with the bubble frequency and also increases with the surfactant concentration. This is attributed to the expedited bubble pressure rise rate due to the increased surfactant dispersion.

\section{ACKNOWLEDGMENT}

This work was supported by the US Department of Energy, Pittsburgh Energy Technology Center (PETC), Contract No. DE-FG22-94PC94120 under the supervision of Dr. Soung S. Kim, Technical Project Monitor at DOE-PETC. The tested coal powder was provided by Mr. David Wildman of DOE-PETC. The tested surfactants and partial financial support were provided from Dr. Paul Berger of Witco Inc. of Houston.

\section{REFERENCES}

1. G. D. Botsaris, and Y. M. Glazman, 1989, Interfacial Phenomena in Coal Technology, Marcel Dekker, New York, Chap. 3.

2. B. W. Brian, and J. C. Chen, 1987, "Surface Tension of Solid-Liquid Slurries," AICHE Journal, Vol. 33, No. 2, pp. 316-318.

3. R. C. Weast (ed.), 1988, CRC Handbook of Chemistry and Physics, CRC Press, Boca Raton, F-34.

4. K. D. Kihm, 1994, "Development and Use of an Apparatus to Measure the Dynamic Surface Properties of Coal-Water Slurry Fuels for Applications to Atomization Characteristics," DOE Report, Contract No. DE-FG-22-92PC92156. 


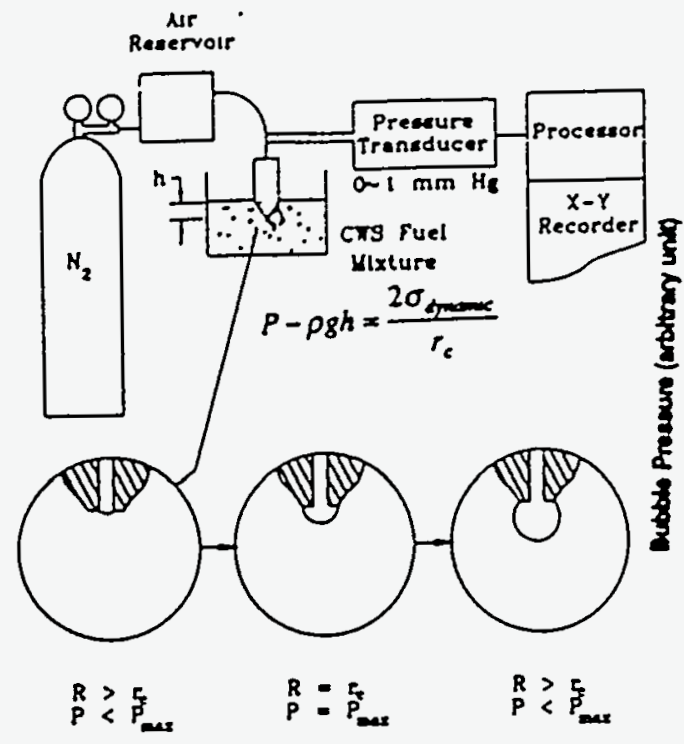

Fig. 1 Schematic illustration of maximum bubble pressure technique.

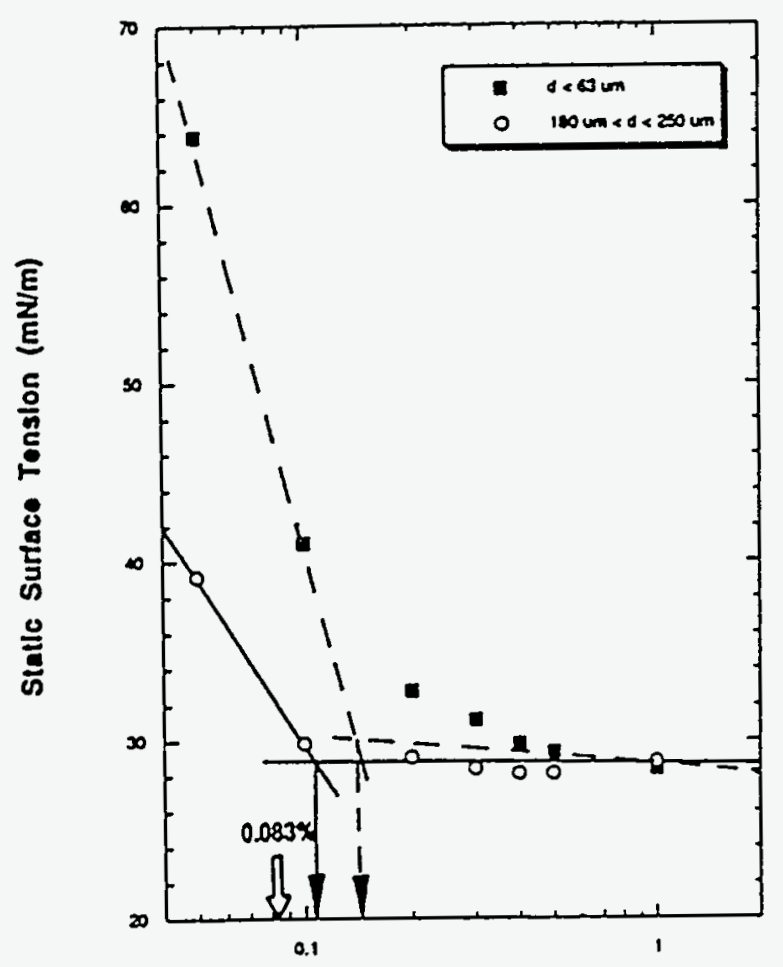

Surfactant Concentration (\%)

Fig. 3 Static surface tension versus concentration of DDBS-soft surfactant for $40 \%$ weight CWS fuels containing different particle sizes.

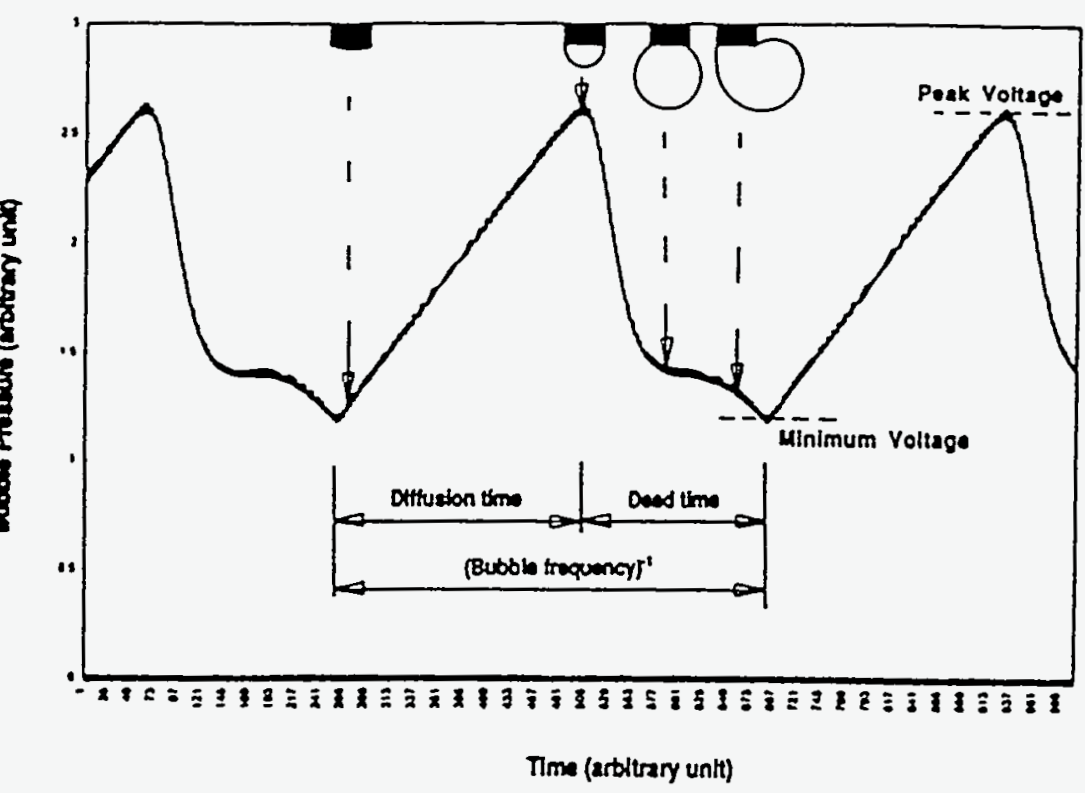

Fig. 2 Temporal history of the bubble pressure.

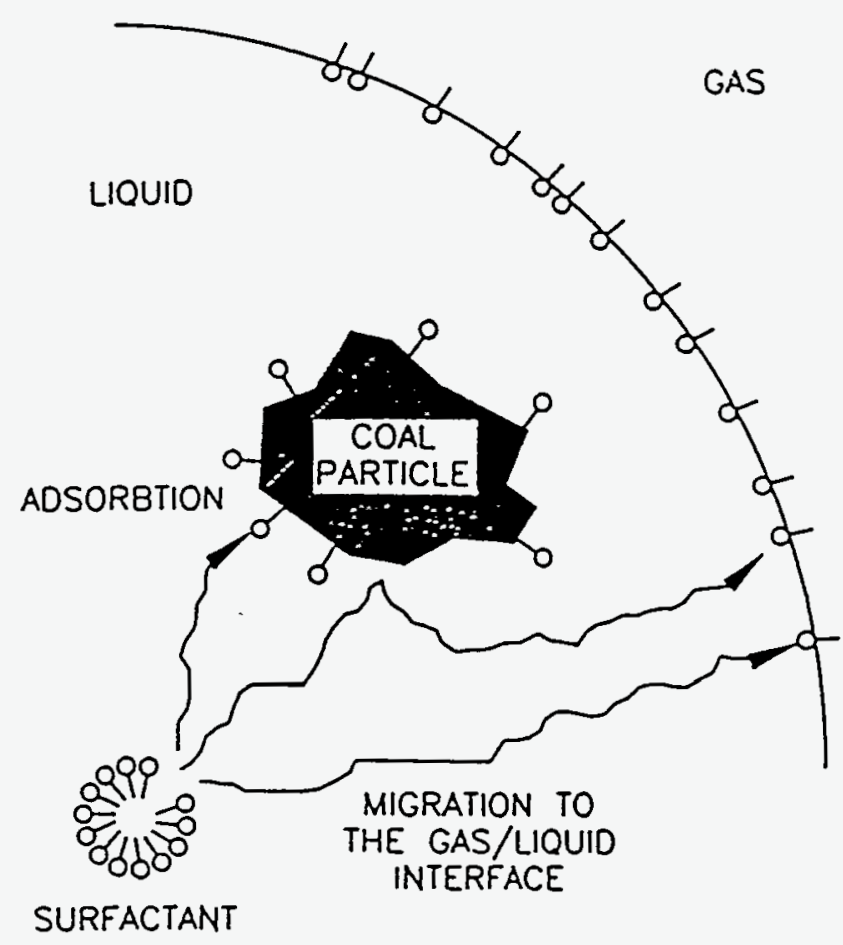

Fig. 4 Schematic illustration of adsorption of surfactant at coal surfaces and physical blocking of surfactant migration by coal paricle. 

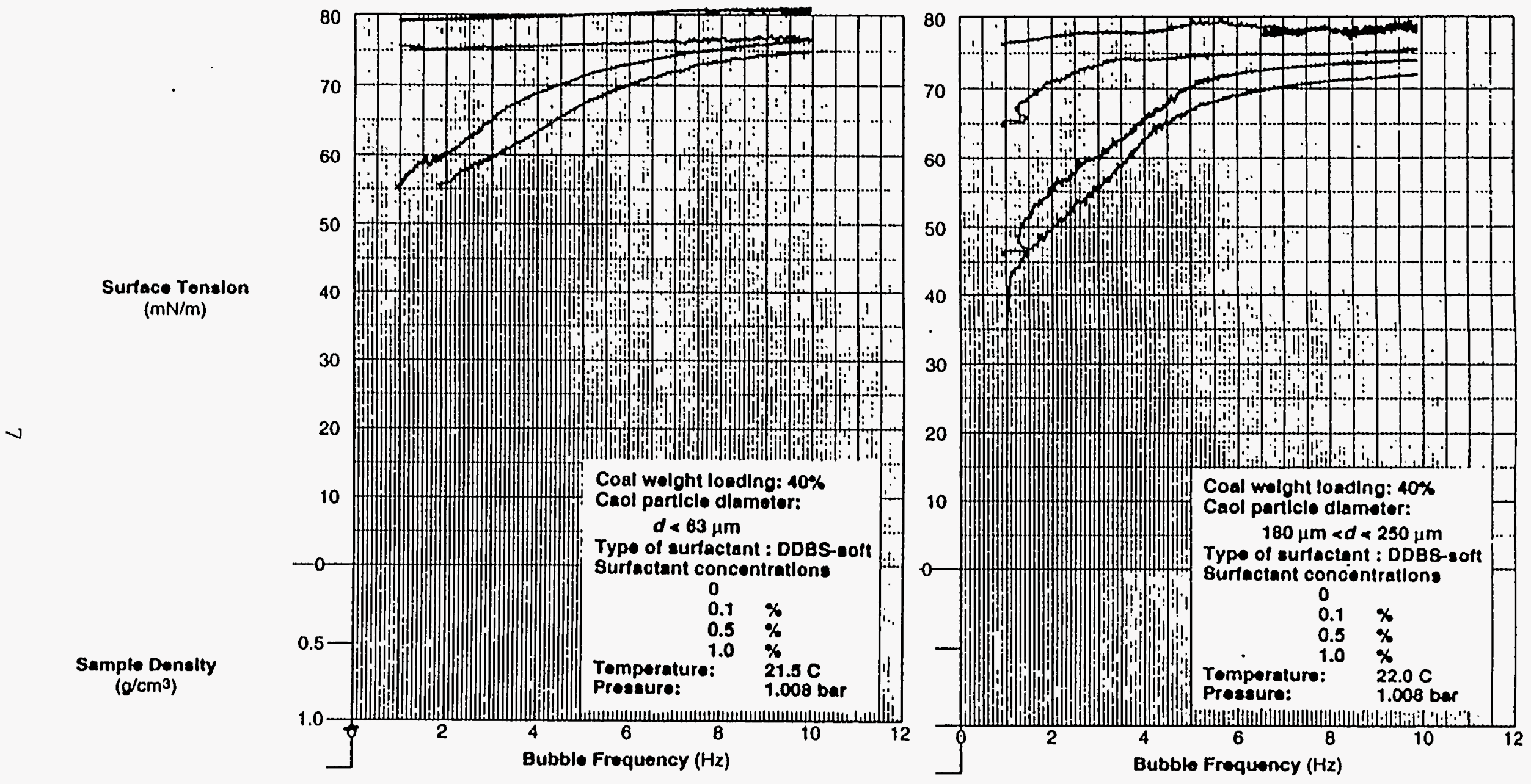

Fig. 5 Dyanmic surface tension versus bubble frequency for different surfactant concentrations. 


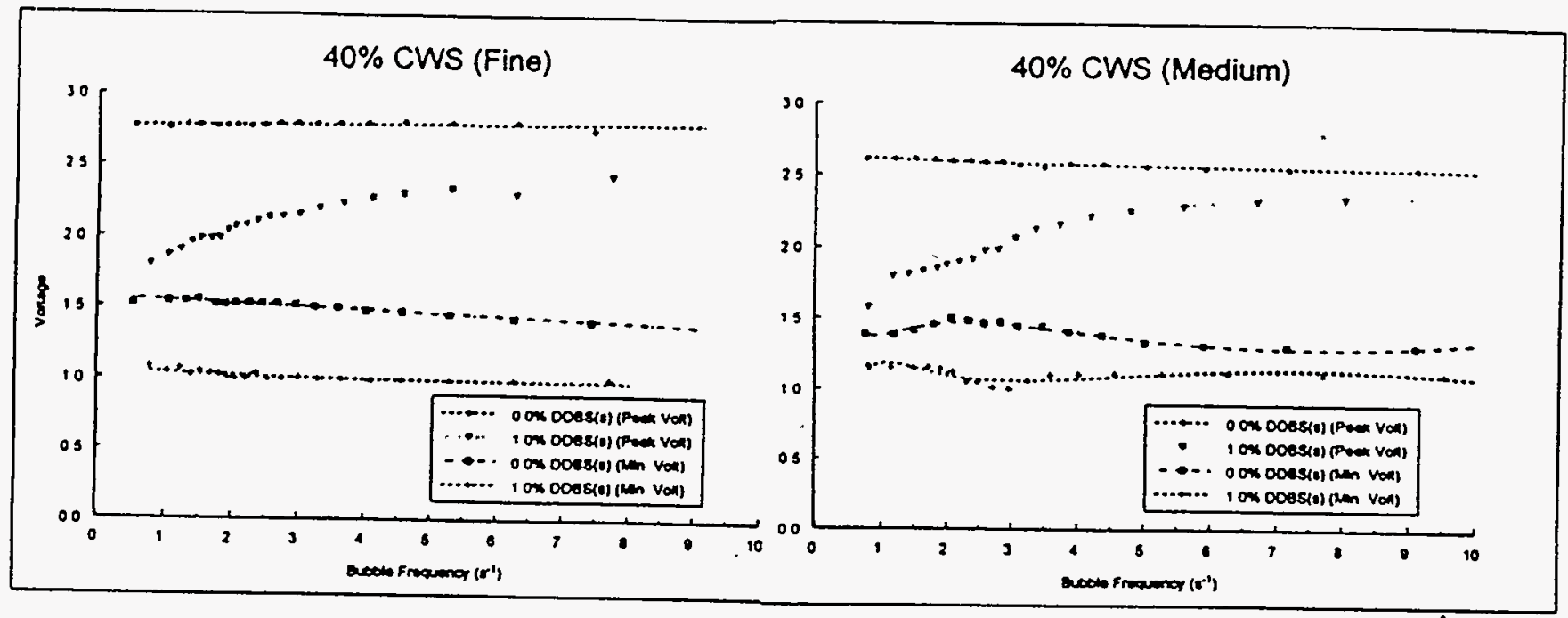

Fig. 6 Peak and minimum voltages versus bubble frequency for (a) fine CWS sample, and (b) coarse CWS sample.

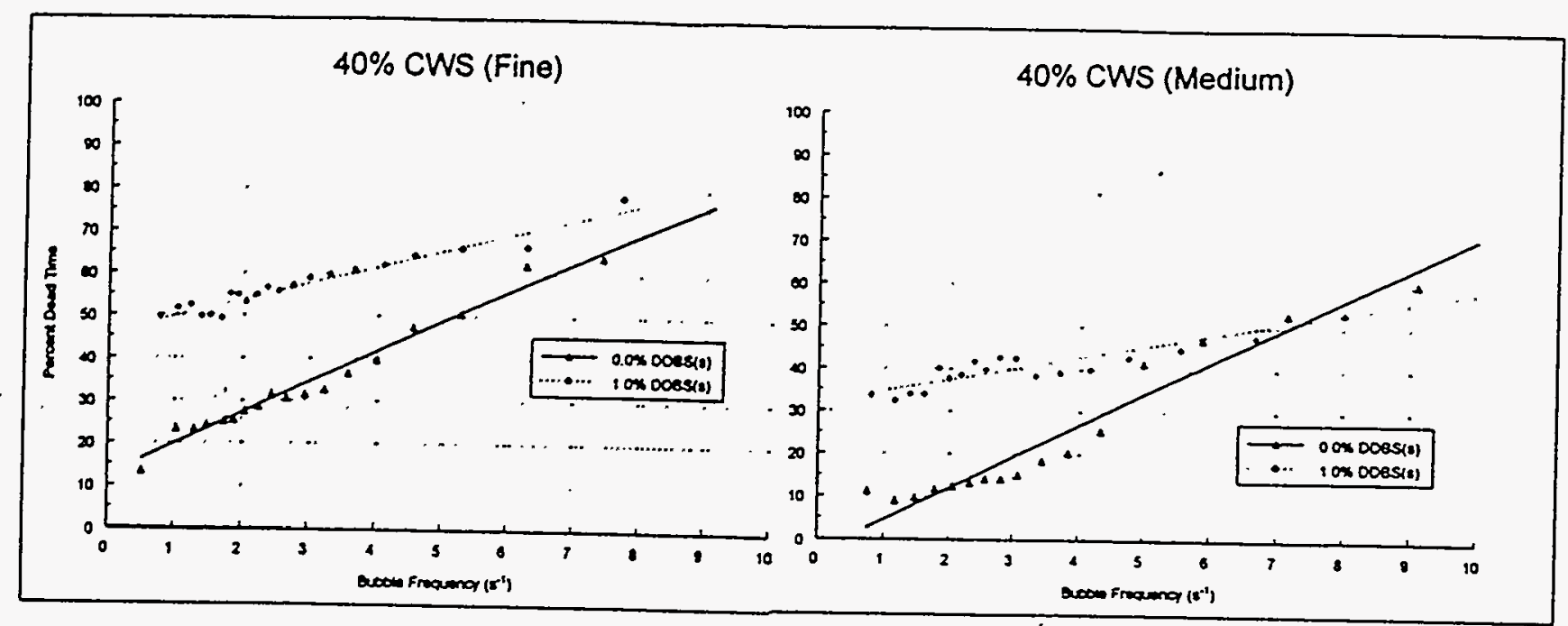

Fig. 7 Percent dead time over the bubble life time versus bubble frequency for (a) fine CWS sample,
and (b) coarse CWS sample. 